\title{
Left-Handed-Media Simulation and Transmission of EM Waves in Subwavelength Split-Ring-Resonator-Loaded Metallic Waveguides
}

\author{
R. Marqués, ${ }^{1, *}$ J. Martel, ${ }^{2}$ F. Mesa ${ }^{3}$ and F. Medina ${ }^{1}$ \\ ${ }^{1}$ Departamento de Electrónica y Electromagnetismo, Universidad de Sevilla, 41012 Sevilla, Spain \\ ${ }^{2}$ Departamento de Física Aplicada 2, Universidad de Sevilla, 41012 Sevilla, Spain \\ ${ }^{3}$ Departamento de Física Aplicada 1, Universidad de Sevilla, 41012 Sevilla, Spain
}

(Received 8 February 2002; published 10 October 2002)

\begin{abstract}
At microwave frequencies, hollow metallic waveguides behave in certain aspects as a "onedimensional plasma." This feature will be advantageously used for simulating the propagation of electromagnetic (EM) waves in left-handed metamaterials provided the hollow waveguide is periodically loaded with split ring resonators. It will be shown that EM transmission in this structure is feasible within a certain frequency band even if the transverse dimensions of the waveguide are much smaller than the associated free-space wavelength. This effect can be qualitatively and quantitatively explained by the left-handed metamaterial theory, thus providing a new experimental validation for such a theory.

DOI: $10.1103 /$ PhysRevLett.89.183901

PACS numbers: 41.20.Jb, 42.70.Qs, 78.20.Ek
\end{abstract}

In 1968 Veselago [1] analyzed electromagnetic (EM) wave propagation through media having simultaneously negative electric permittivity and negative magnetic permeability. Since the $\mathbf{E}, \mathbf{H}$ fields and the wave vector $\mathbf{k}$ of a propagating plane EM wave form a left-handed system in these materials, Veselago referred to them as "lefthanded" media. Other interesting properties of such media are, among others, negative refractive index and reversed Doppler effect [1]. The nonexistence of transparent left-handed media in Nature made Veselago's analysis and predictions remain for a long time as a mere theoretical curiosity. However, recently, microwave propagation through an artificial left-handed medium (or metamaterial) has been demonstrated by Smith et al. [2]. The left-handed metamaterial reported in this paper was, in fact, the superposition of two different artificial media: a two-dimensional plasma working below its plasma frequency and an artificial negative magnetic permeability medium (NMPM). Artificial plasmas are well-known materials that can be realized, for instance, by using a regular array of conducting wires [3]. Artificial NMPM have recently been proposed by Pendry et al. [4] and experimentally demonstrated by Smith et al. [2]. These latter media are composed of electrically small resonant particles, which show a very high diamagnetic susceptibility above and around its resonance frequency. When these particles are arranged in a regular lattice and illuminated in a proper way, their high diamagnetic susceptibility causes the resulting composite medium to have a negative effective magnetic permeability within a certain frequency range. In this range the resulting artificial medium is called an artificial NMPM. The split ring resonator (SRR) proposed by Pendry et al. [2,4] (see Fig. 1) fulfills all the requirements for the design of the aforementioned NMPM.

The artificial plasma medium proposed in [2] was a simulated two-dimensional plasma, made by placing a regular array of metallic posts between two parallel metallic plates. Nevertheless, a significant simplification of the plasma simulation is possible by using a hollow metallic waveguide, as suggested by the dispersive behavior of these waveguides. It is well-known that hollow metallic waveguides can support TE and TM modes satisfying the following general dispersion relation [5]:

$$
k=\omega \sqrt{\mu_{0} \epsilon_{\mathrm{eff}}},
$$

where $k$ is the propagation constant, $\omega$ the angular frequency, $\mu_{0}$ the magnetic permeability of vacuum, and $\epsilon_{\text {eff }}$ an effective dielectric constant given by

$$
\epsilon_{\mathrm{eff}}=\epsilon_{0}\left(1-\frac{\omega_{0}^{2}}{\omega^{2}}\right)
$$

with $\omega_{0}$ being the cutoff frequency for the considered mode and $\epsilon_{0}$ the dielectric permittivity of vacuum. Considering now that the dispersion relation (1) and (2)

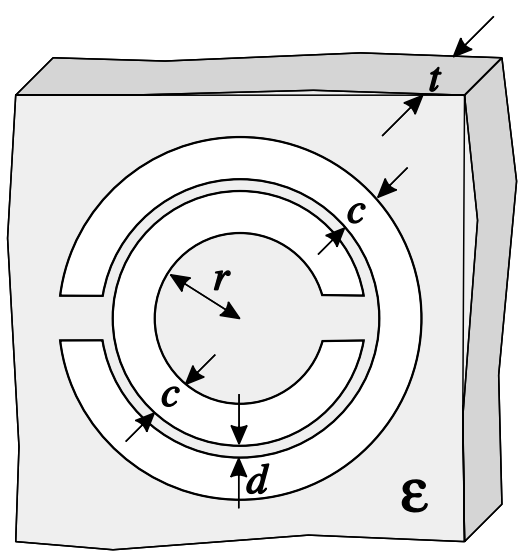

FIG. 1. The split ring resonator (SRR) used in this work. Structural parameters are $r=1.4 \mathrm{~mm}, c=0.5 \mathrm{~mm}, d=$ $0.2 \mathrm{~mm}, t=0.49 \mathrm{~mm}$, and $\epsilon=2.43 \epsilon_{0}$. 
is identical to that of a lossless plasma with plasma frequency $\omega_{0}$, a hollow metallic waveguide can be regarded as a "one-dimensional plasma" with respect to the EM propagation along the axial direction. If a square waveguide was used (provided that the direction of propagation of the EM wave is restricted to be parallel to the waveguide axis), a one-dimensional plasma isotropic in the transverse plane could then be simulated.

Once postulated the square waveguide can model a one-dimensional plasma, this reasoning can be extended to predict that the behavior of a EM wave in a left-handed material can be simulated by placing a periodic array of SRRs inside the square waveguide. The role of the SRR particles is to introduce the effective negative permeability necessary to obtain the left-handed behavior. To keep the modeling of the system as simple as possible and to enhance the interaction SRR-EM field, the SRR array should be placed at the plane of symmetry of the square waveguide, with a spacing between the SRRs equal to the waveguide lateral side walls dimension (see Fig. 2 for a sketch of this setup). Clearly, the operation frequency must be below the cutoff frequency of the dominant mode of the hollow waveguide in order to simulate a medium with negative dielectric permittivity. Following the above rationale, it is expected that EM transmission along the waveguide at cutoff (i.e., in the plasma below the plasma frequency) takes place only at that frequency range where negative permeability coexists with negative permittivity. This "left-handed passband" should coincide with the frequency band at which the composite SRRs and waveguide device simulates a left-handed metamaterial.

The experimental setup shown in Fig. 3 was designed and built in order to validate the above hypothesis. The left-handed medium is simulated by a regular array of SRRs placed at the half-plane of a square waveguide of $6 \mathrm{~mm}$ width (cutoff frequency at $25 \mathrm{GHz}$ ). The spacings between the SRR centers is also set to $6 \mathrm{~mm}$, and the SRR dimensions are specified in Fig. 1. Thus, the SRR-loaded square waveguide simulates an infinite one-dimensional left-handed metamaterial whose lattice constant is $6 \mathrm{~mm}$. Three square waveguide sections with lengths $l=12,24$, and $36 \mathrm{~mm}$ were fabricated in order to investigate the effect of the finite size of the simulated left-handed

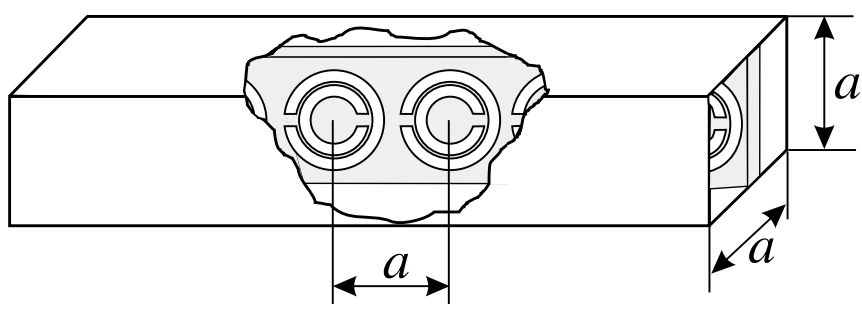

FIG. 2. The SRR-loaded square waveguide $(a=6 \mathrm{~mm})$ used for simulating the left-handed metamaterial. medium along the longitudinal direction. The SRRs are printed on a commercial substrate of dielectric constant $\epsilon=2.43 \epsilon_{0}$ and thickness $t=0.49 \mathrm{~mm}$. The SRR-loaded square waveguide is placed between two sections of commercial coaxial to rectangular waveguide transitions (the rectangular waveguide is a WR137 waveguide of $35 \times$ $16 \mathrm{~mm}^{2}$ cross section and cutoff frequency at $4.3 \mathrm{GHz}$ ). In order to facilitate the excitation of the first and the last SRRs in the array, they were partially placed out from the square waveguide. Therefore, the three sections of square waveguide with lengths $l=12,24$, and $36 \mathrm{~mm}$ will host, respectively, three, five, and seven SRRs. However, they simulate only two, four, and six unit cells of the lefthanded medium. The automatic network analyzer HP8510 -B is used to measure the transmission coefficient $\left(\left|S_{12}\right|\right)$ through the three sections of loaded waveguides already described.

An electromagnetic model, whose details are discussed elsewhere [6,7], has been used to obtain the macroscopic parameters of the simulated left-handed medium. This simulation assumes that the effective dielectric constant of the medium is given by (2), with $\omega_{0}$ being the cutoff frequency of the hollow square waveguide of Fig. 2 $(\sim 25 \mathrm{GHz})$. The effective magnetic permeability is taken as that of an infinite cubic array of SRRs with lattice constant $a=6 \mathrm{~mm}$ (the SRRs spacing and the lateral side wall dimensions in the waveguide). With regard to the SRR medium, the model follows the guidelines reported in [6], although the inductance of the SRR particles - which appears as an adjustable parameter in [6] - is now computed in a self-consistent way by using the quasistatic model reported in [7]. The results of this simulation for the normalized phase constant of a transverse EM wave in the assumed left-handed continuous medium are shown in Fig. 4. The resonance frequency of the SRRs, which approximately coincides with the onset of the left-handed passband, was predicted at about $6 \mathrm{GHz}$, well below the cutoff frequency of the square waveguide $(25 \mathrm{GHz})$. The predicted bandwidth of the lefthanded passband is about $400 \mathrm{MHz}$. The same model provides the phase constant for propagation in an infinite cubic array of SRRs of lattice parameter $a=6 \mathrm{~mm}$ by simply equating the effective permittivity to $\epsilon_{0}$. The

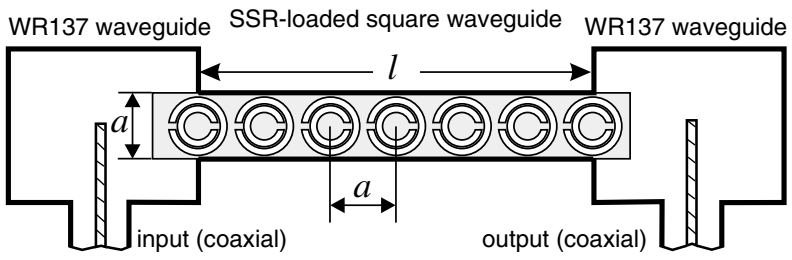

FIG. 3. Sketch of the experimental setup. The microwave signal is fed and received by means of two standard coaxial to rectangular waveguide junctions. Three lengths for the SRRloaded waveguide section are considered in the experiments: $l=12,24$, and $36 \mathrm{~mm}$. 


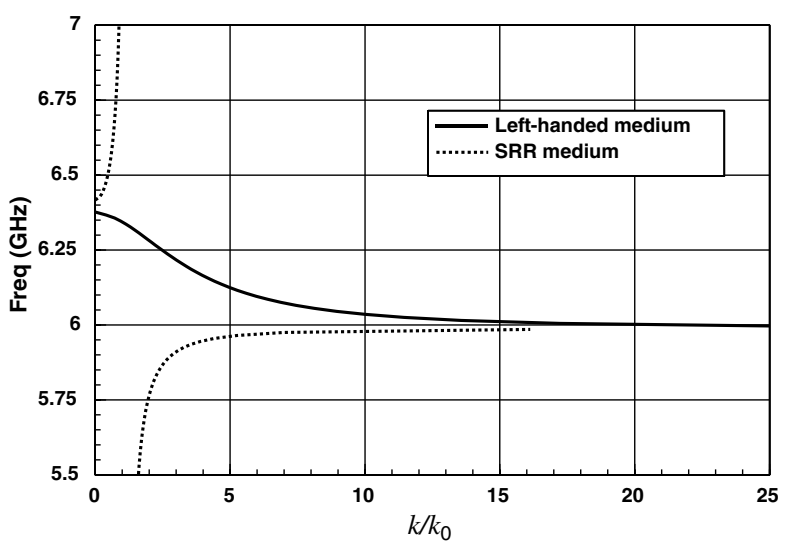

FIG. 4. Computed values for the normalized (to the freespace wave number, $k_{0}$ ) phase constants of the simulated left-handed and the SRR media. The lattice constant is $a=$ $6 \mathrm{~mm}$ and the SRR parameters are those of Fig. 1. The plasma frequency is the cutoff frequency of the dominant $\mathrm{TE}_{10}$ mode of the square hollow waveguide in Fig. 2.

results for this normalized phase constant are also shown in Fig. 4. As expected, a forbidden band appears for the SRR medium, which almost coincides with the passband of the composite media (the small mismatch is due to bianisotropic effects [6]).

The results for the experimental setup of Fig. 3 are shown in Figs. 5(a)-5(c). In all cases a similar frequency passband was measured around and above $6 \mathrm{GHz}$, the predicted frequency, with a neat transmission region of more than $60 \mathrm{~dB}$ above the noise floor. The measured bandwidth of the passband is also very close to that obtained from the theoretical model. It should be noticed that the $\left|S_{12}\right|$ level inside the transmission region is approximately the same for the three different-length waveguide sections considered. This fact ensures that the insertion loss of the structure is not associated with the transmission along the three different waveguide sections but to the reflections at the waveguide transitions, which were not adapted in our experiment. It can then be admitted that all three SRR-loaded waveguide sections simulate the same (almost lossless) effective medium, apart from second order effects related to truncating the medium along the axial direction. In the $\left|S_{12}\right|$ plots of Fig. 5 several small subpeaks appear in the passband, which can be attributed to the relatively small number of SRR particles along the axial direction (note that the number of subpeaks increase with the number of resonators). A simple inspection of the sequence of curves in Fig. 5 suggests that the curve in the transmission region would get smoother if many resonators were used. Clearly, in such a case, the continuous medium description would be more accurate.

The good agreement between the experiments and the predictions of the proposed theoretical model is a strong argument supporting our initial hypothesis. In addition, a similar rationale as that in [2] can also be argued to sustain the left-handed nature of the experimental passband reported in Figs. 5(a)-5(c). Let us consider the twodimensional array of SRR-loaded waveguides shown in Fig. 6 (with the dimensions specified in Fig. 2). If the SRRs are removed, it is well known [5] that the remaining mesh of metallic plates support a fundamental TE mode of propagation. This mode shows an effective permittivity given by (2), with $\omega_{0}$ being the cutoff frequency of the hollow square waveguide of Fig. $2(25 \mathrm{GHz})$. Considering that the average fields in each waveguide cross section are perpendicular to the direction of propagation and provided that all TE waves in all the waveguides of Fig. 6 are equally polarized, it can be deduced that this structure actually simulates a plasma whose
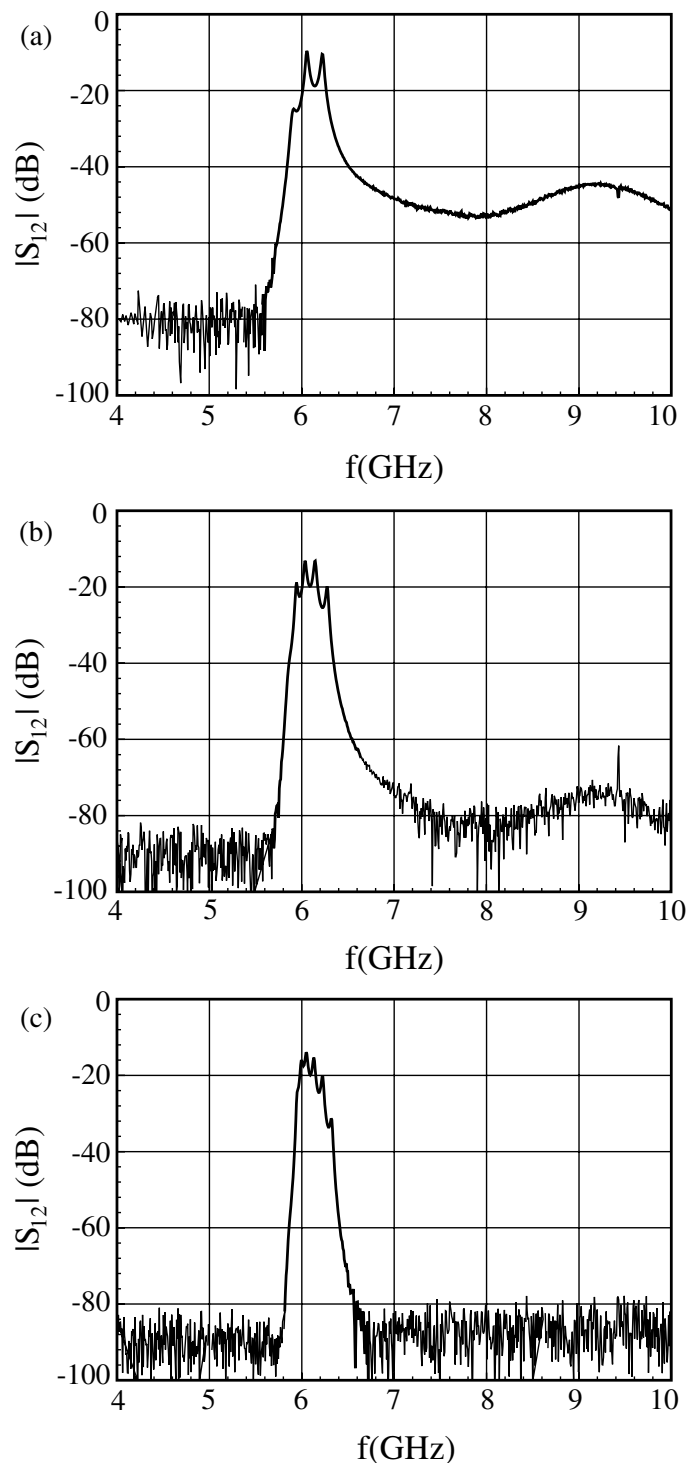

FIG. 5. Measured transmission coefficient $\left(\left|S_{12}\right|\right)$ for the experimental setup in Fig. 3. Three different lengths of the SRRloaded square waveguide are studied: (a) $l=12 \mathrm{~mm}$, (b) $l=24 \mathrm{~mm}$, and (c) $l=36 \mathrm{~mm}$. 


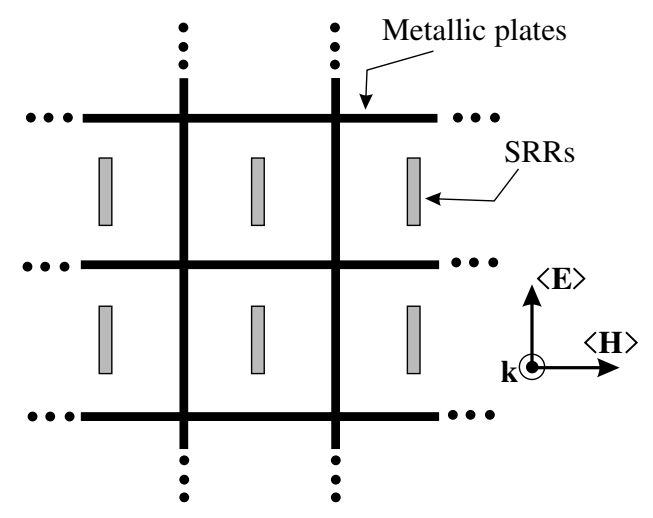

FIG. 6. An infinite array of SRR-loaded square waveguide. The polarization of the left-handed wave propagating in this structure is also shown.

dielectric permittivity is negative for all the frequency range considered in our experiment. If, on the contrary, the metallic plates are removed from Fig. 6, the remaining medium is an infinite cubic array of SRRs. For the considered mean field polarization (see Fig. 6), this medium is known to behave as an NMPM within certain frequency range $[2,4]$, showing a forbidden frequency band in this range of frequencies (this latter result has been proven experimentally in [2]). Since the phase constant for the SRR medium as well as the aforementioned forbidden frequency band are properly predicted and computed from the left-handed metamaterial theory (see Fig. 4), following the same rationale as in [2], it can be concluded that the passband appearing in Figs. 5(a)-5(c) is due to the simultaneous presence of negative effective permeability and permittivity in the structure of Fig. 6 (and in the one-dimensional simulation of Fig. 2).

As any hollow metallic waveguide shows the dispersion relation summarized in Eqs. (1) and (2), the consideration of such waveguides as one-dimensional plasmas, if correct, goes beyond the particular case of a square waveguide. A similar behavior would be expected for any SRR-loaded metallic waveguide. Thus Fig. 7 shows the results of an experiment similar to that in Fig. 5(c), but replacing the square waveguide by a cylindrical waveguide with an internal diameter of $6 \mathrm{~mm}$. The similarity between the results in Figs. 5(c) and 7 is apparent. From this perspective, it should be highlighted that the reported experiments have shown the possibility of EM transmission through a waveguide operating far below the cutoff frequency of its dominant mode (subwavelength operation) by inserting periodically distributed SRRs. It has also been shown that the left-handed metamaterial theory predicts and explains this nonintuitive result (it is known that EM wave propagation in hollow

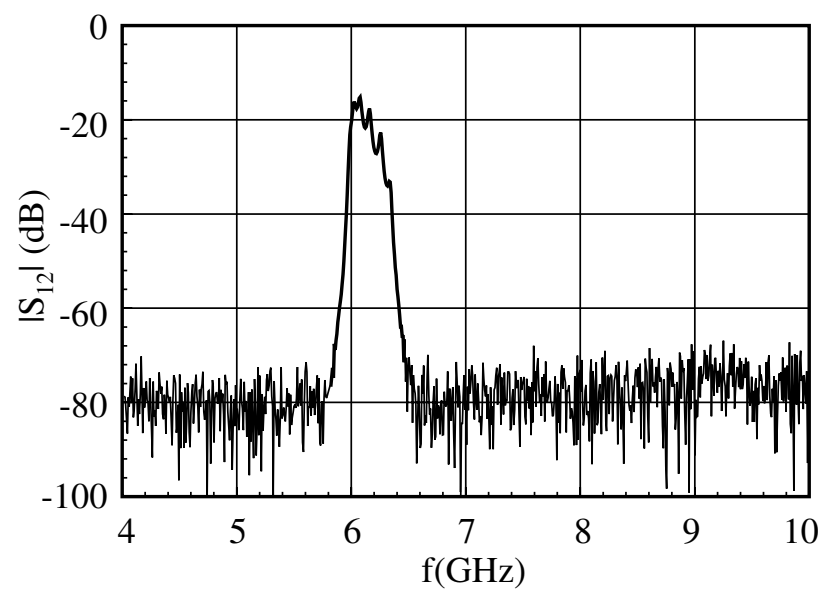

FIG. 7. Measured transmission coefficient $\left(\left|S_{12}\right|\right)$ for the experimental setup in Fig. 3 but using now a cylindrical waveguide with $6 \mathrm{~mm}$ of diameter and $l=36 \mathrm{~mm}$.

metallic waveguides takes place only when the transverse waveguide dimensions are of the order of the free-space wavelength [5]). Possible applications of this effect in the design of new types of small-size waveguides, filters, delay lines, and other microwave and millimeter-wave devices can be expected. As far as the transmission of EM waves through subwavelength SRR-loaded metallic waveguides is suggested and accurately predicted by the theory of left-handed metamaterials, these applications could also be considered as new applications of this recently developed theory.

This work has been supported by the Spanish Ministry of Science and Technology and FEDER funds (Project No. TIC2001-3163).

*Electronic address: marques@us.es

[1] V. G. Veselago, Sov. Phys. Usp. 10, 509 (1968).

[2] D. R. Smith, W. J. Padilla, D. C. Vier, S. C. Nemat-Nasser, and S. Schultz, Phys. Rev. Lett. 84, 4184 (2000).

[3] J. M. Pitarke, F. J. García-Vidal, and J. B. Pendry, Phys. Rev. B 57, 15261 (1998).

[4] J. B. Pendry, A. J. Holden, D. J. Robbins, and W. J. Stewart, IEEE Trans. Microwave Theory Tech. 47, 2075 (1999).

[5] J. D. Jackson, Classical Electrodynamics (Wiley, New York, 1999), 3rd ed.

[6] R. Marqués, F. Medina, and R. Rafii-El-Idrissi, Phys. Rev. B 65, 144440 (2002).

[7] R. Marqués, F. Medina, F. Mesa, and J. Martel, in Advances in Electromagnetics of Complex Media and Metamaterials, edited by S. Zouhdi, A. Shivola, and M. Arsalane (Kluwer, Dordrecht, 2002), p. 123. 